\title{
Ovarian Endometrioid Adenocarcinoma
}

National Cancer Institute

\section{Source}

National Cancer Institute. Ovarian Endometrioid Adenocarcinoma. NCI Thesaurus. Code C7979.

An endometrioid adenocarcinoma arising from the ovary. It comprises $10 \%$ to $25 \%$ of all primary ovarian carcinomas. Grossly, endometrioid carcinoma may present as a cystic or solid mass. Microscopically, the tumor greatly resembles the appearance of the ordinary type of endometrial adenocarcinoma. As a group, endometrioid carcinoma has a prognosis twice as good as that of serous or mucinous carcinoma. 